\title{
La crianza natural: una solución biográfica frente a la desfamiliarización y mercantilización del cuidado
}

\section{Leila Abdala}

Universidad Nacional del Litoral

leilabdala68@gmail.com

RESUMEN

Este articulo se propone explorar los significados y prácticas maternales asociados al estilo de crianza que las mujeres estudiadas definen como «natural», mediante la descripción del repertorio de prácticas maternales de cuidado que supone, el análisis de las situaciones típicas de cuidado que se derivan de ella y la identificación del rol que tienen los varones en este estilo de crianza desde la mirada de las mujeres-madres. Se vale de una etnografía de rondas de lactancia y crianza llevada a cabo en la ciudad de Santa Fe (Argentina), donde se recolectaron tres tipos de información: notas de campo sobre los encuentros en talleres, entrevistas semiestructuradas con madres, y seguimiento de los documentos y discursos públicos de los grupos dedicados al tema.

Reuniendo los hallazgos de nuestro estudio, sostenemos que, frente a un contexto institucional de mercantilización de la vida intima (Hochschild, 2008), las formas de vivir la maternidad de las mujeres estudiadas, y en especial las demandas por una «crianza natural», pueden ser comprendidas como una solución biográfica a la desfamiliarización y mercantilización del cuidado de los niños pequeños. El retorno al hogar para el cuidado de los niños pequeños adquiere una valoración positiva en mujeres de clases medias profesionalizadas que participan de los espacios y talleres estudiados.

Palabras clave: maternidad, crianza natural, situaciones de cuidado, mercantilización del cuidado. 


\section{Natural parenting: a biographical solution to the defamiliarization and commodification of care}

\section{ABSTRACT}

The article explores meanings and maternal practices associated with the parenting style that middle class professional women define as "natural". It describes the repertoire of maternal care practices that this parenting style implies, and analyzes the typical care situations that derive from it. Also, it identifies the role that men play in this parenting style from the perspective of female-mothers. The article is based on an ethnographic study of parenting and breastfeeding groups, carried out in Santa Fe city (Argentina). It adopted various techniques for data collection: field notes from workshop meetings, semi-structured interviews with mothers, and the followup of the documents and public speeches of the groups dedicated to research this topic.

According to our findings, we maintain that the ways in which the women of professionalized middle classes who participate in the study live their motherhood and the demands for a "natural upbringing" in the context of commercialization of human care (Arlie Hochschild, 2008) can be understood as a biographical solution to the defamiliarization and commercialization of the care of young children. We maintain also that returning home for child-care acquires positive assessment among them.

Keywords: motherhood, natural upbringing, care situations, commodification of care. 


\section{INTRODUCCIÓN}

Una de las grandes trabas para abrirse en el parto y para entregarse en la crianza tiene que ver con no aflojar. De decir «eso es de las mujeres de antes, de mi abuela. Yo ahora me dedico a mí y eso es lo que las mujeres conseguimos con tanta cosa», y quizás es no dejarse sentir. Es como que, por un lado, la maternidad te dice «acá está tu lugar» y tu conciencia y cabeza dice «no, yo logré esto, llegar acá». Y las mujeres la pasan mal, o no, los niños que se la pasan con huevitos, chupete, niñera... Me da lástima que se identifique tanto la maternidad con eso negativo. Si se dejaran sentir más podrían hacer las dos cosas. No verlo como la maternidad o la libertad. La maternidad me abre. Yo quizás hubiese seguido siendo docente, o no. Hay mamás que no pueden ver las necesidades de su hijo. También siento que estamos en un camino y cada vez más iremos encontrándonos. Yo puedo elegir cuando estoy empoderada; si no, es un mandato más: ¿vuelvo al trabajo porque quiero o porque debo? (fragmento entrevista Florencia).

La socióloga y feminista Arlie Russell Hochschild inicia su libro La mercantilización de la vida íntima. Apuntes de la casa y el trabajo (2008) preguntándose acerca de qué brújula oculta en su vida personal explica su profundo interés con relación a sus estudios sobre la emoción, el género, la familia, el capitalismo y la globalización. Para comenzar a esbozar una respuesta a tan inquietante pregunta que vincula su trayectoria individual con su interés intelectual, nos relata las imágenes del recuerdo que tiene de su madre, ama de casa de tiempo completo: «en la mirada de mi madre había un dejo de tristeza [...] ella miraba al mundo con ojos melancólicos y sombríos» y —en su opinión — no se veía tan feliz como su padre: varón universitario y diplomático, quien silbaba alegremente al bajar los peldaños de la casa de dos en dos cuando salía todas las mañanas a trabajar. «Mi madre era la persona triste que nos cuidaba y mi padre la persona alegre que no nos cuidaba» (Hochschild, 2008, pp. 15-16). La autora reflexiona que 
desde pequeña asumió la idea de que las tareas del cuidado no necesariamente producen felicidad en las personas que las ejercen.

Como muchas otras mujeres estadounidenses de su generación, blancas y de clase media, en la década de 1960, Hochschild migró de la cultura emocional ${ }^{1}$ de su madre a la de su padre «sin obtener ciudadanía en ninguna de las dos». La autora plantea aquí lo que nosotras entendemos como un primer movimiento: la migración de mujeres del mundo femenino - que tiene al cuidado como valor sacro - al mundo masculino - con un fuerte culto al trabajo fuera del hogar-, mediante una asimilación o aculturación de estas al mundo de las reglas masculinas. Es decir, mujeres que, a diferencia de sus madres, se sumergieron —no sin conflictos y tensiones - en el mercado laboral, con la expectativa de encontrar allí, a través de altas inversiones emocionales en ese espacio, una fuente de reconocimiento social, de estatus vinculado con la vida profesional en términos inversamente proporcionales al reconocimiento otorgado por el trabajo sobre los cuidados en la casa. Este «movimiento» tuvo como efecto no buscado lo que la autora denomina «el espíritu mercantil de la vida íntima», producto de una afinidad electiva entre el ethos feminista y el ethos mercantil. La autora arriesga la siguiente hipótesis: la ética protestante es al capitalismo lo que el feminismo es a la mercantilización de los cuidados. Para ella, el feminismo abrió el camino para que las mujeres habiten la vida pública mediante el supuesto ideológico de la igualdad entre los géneros $\mathrm{y}$, como consecuencia no buscada ${ }^{2}$, sentó las bases del espíritu mercantil de la vida misma que trae consigo, entre otras cosas, la mercantilización de los cuidados.

Esta hipótesis cobra validez cuando observamos la manera en la cual, en las últimas décadas, diversas instituciones, bajo la luz de saberes autorizados y expertos - mediatizados por el mercado-, han ido colonizando los aspectos vinculados con el cuidado, escolarización, salud, recreación, nutrición, etc. de

1 «Una cultura emocional es un conjunto de rituales, de creencias en torno de los sentimientos y reglas directrices de los sentimientos que inducen a focalizar las emociones e incluso inspiran un sentido de lo "sagrado" que selecciona algunos vínculos sociales y los prioriza sobre los otros [...] Toda cultura emocional genera sus propios patrones de sacralidad» (Hochschild, 2008, p. 293).

2 Al igual que Calvino, las fundadoras del feminismo se habrían preocupado si hubieran imaginado las tendencias culturales que se entretejerían alrededor de sus ideales básicos. «La igualdad está bien — habrían dicho quizá si vivieran hoy en día-, pero no tenemos por qué permitir que la cultura capitalista establezca la base cultural de ese logro» (Hochschild, 2008, p. 41). 
los niños, interviniendo estos sistemas activamente en momentos claves de las biografías de las personas, como el nacimiento, el embarazo y la crianza.

Sin embargo, como la autora nos alerta, es posible encontrar oposiciones a estas tendencias y procesos. A la luz de los primeros hallazgos del trabajo empírico realizado en la tesis doctoral, nos arriesgamos a esbozar la siguiente pregunta: ¿no es posible pensar en un «segundo movimiento» capaz de ser definido como la «crítica a la mercantilización y desfamiliarización de los cuidados»? ¿Es posible que el cuidado (re)cobre valor y reconocimiento en algunos grupos de mujeres de clases medias profesionalizadas? ¿Estamos en presencia de un desplazamiento del patrón de sacralidad que va del trabajo profesional al cuidado? La conjetura es la siguiente: mujeres jóvenes de clases medias profesionalizadas ya socializadas en el «mundo masculino», cuyas madres - a lo Hochschild — han migrado en sus propias biografías de un mundo a otro, reivindican una vuelta al mundo familiar de los cuidados, donde encuentran una gran fuente de reconocimiento social. La escena a problematizar es la siguiente: un grupo de mujeres jóvenes de clases medias desestima el reconocimiento social de su trayectoria profesional para buscarlo y encontrarlo en el cuidado de sus hijos pequeños. Mientras sus contemporáneas se benefician de las niñeras, los servicios de cuidados extendidos y las mamaderas, estas madres rechazan todo lo que perciben como mecanismos de separación madre-hijo.

Con estas preguntas como punto de partida, en este artículo se exploran los significados y prácticas maternales asociados al estilo de crianza que las mujeres analizadas definen como «natural» o de «apego», mediante: (i) la descripción del repertorio de prácticas maternales de cuidado que supone; (ii) el análisis de las situaciones típicas de cuidado que se derivan de ella, y (iii) la identificación del rol que tienen los varones en este estilo de crianza desde la perspectiva de las mujeres. El texto se estructura a partir del abordaje de cada uno de esos objetivos.

El análisis propuesto se basa en una etnografía de rondas de lactancia y crianza, llevada a cabo en la ciudad de Santa Fe (Argentina). Se realizaron entrevistas semiestructuradas a doce madres que participan activamente de grupos de reflexión sobre embarazo, parto y crianza. Ellas tienen entre 21 y 36 años, se identifican como heterosexuales, y al momento de las entrevistas se encontraban en un momento del ciclo de vida del hogar y familiar muy específico: el de la crianza de niños pequeños. En su mayoría, poseen títulos universitarios o se encontraban estudiando alguna carrera de grado y conviven con los padres de sus hijos. También recolectamos discursos públicos de las organizaciones y grupos, y realizamos una observación participante en talleres de preparación para el parto 
y nacimiento, grupos de crianza y apoyo a la lactancia materna. Los espacios seleccionados para realizar las observaciones fueron tres instituciones de la ciudad que son reconocidas públicamente por la promoción de crianzas naturales y partos humanizados, las cuales concebimos como espacios privilegiados de producción de sujetos-madres bajo la socialización de una serie de habilidades maternales, consejos y «reglas del sentir» (Hochschild, 2008; Illouz, 2007, 2010).

El estudio sobre este estilo de crianza problematiza enfoques teóricos y posiciones feministas que, por un lado, conciben la función materna como alienante $\mathrm{o}$ - en palabras de Badinter (2011) — como «una nueva forma de esclavitud $»^{3}, \mathrm{y}$ por otro, reclaman la función materna como una fuente de placer, conocimiento y empoderamiento ${ }^{4}$. Nuestra perspectiva, en cambio, se distancia de paradigmas normativos para indagar en los significados y prácticas que se ponen en juego a la hora de ejercer la maternidad, buscando arrojar luz sobre las paradojas emergentes en las múltiples formas de vivenciarla.

\section{LA CRIANZA NATURAL: UN REPERTORIO DE «BUENAS» PRÁCTICAS MATERNALES DE CUIDADO}

Antes de comenzar con la descripción de las prácticas maternales asociadas a la crianza natural, creemos necesario inscribir este estilo de crianza dentro de un proceso en el cual la infancia se ha constituido como una identidad social propia (Segalen, 2013). Como se reconoce a partir del análisis histórico de Philippe Ariès ${ }^{5}$

3 De Beauvoir (1977 [1949]) fue una de las primeras en concebir la maternidad como una actividad alienante que limita a las mujeres impidiéndoles cumplir con su destino más trascendente: mientras son reproductoras no hacen más que reproducir el orden social existente. En este sentido, para ella, en la renuncia a ser madres estaría la posibilidad de su igualdad con los varones, la oportunidad de desligarse de su potencia reproductora para aventurarse, por fin, a su potencia creadora. En la década de 1970, Firestone (1970) — otra de las referentes del feminismo de la igualdad o del feminismo radical — sugirió que la vinculación problemática entre el binomio mujer-naturaleza podía ser disuelta a través de la anticoncepción, pero también de la reproducción artificial.

4 Esta perspectiva feminista propone asumir la capacidad generadora del cuerpo de las mujeres y convierte la maternidad en sinónimo de un vínculo intrínseco y básico entre las mujeres; contempla con un nuevo enfoque las relaciones materno-filiales (Irigaray, 1992; Sau, 2013) y, al mismo tiempo, rechaza la «institución materna» (Rich, 1976). Estas autoras identificadas dentro del «feminismo de la diferencia», también llamado «feminismo maternal», opinan que la experiencia de las mujeres como madres les da una capacidad moral superior porque está relacionada con una serie de valores más solidarios y humanizados (Chodorow, 1984).

5 En su obra L'enfant et la vie familiales sous l'Ancien Régime («El niño y la vida familiar bajo el Antiguo Régimen»), publicada en 1960, Ariès señala que, a partir de un período que puede 
y Norbert Elias ${ }^{6}$, desde la modernidad la infancia pasó a ser centro de atención de la familia y las instituciones educativas. Sin embargo, es a partir de las últimas décadas ${ }^{7}$ cuando el movimiento de individualización que opera en la sociedad, luego de haber beneficiado a las mujeres, concierne también al niño (Segalen, 2013). Este no es ya un ser sobre el cual hay que imprimir las tradiciones familiares y a quien es preciso imponerle la autoridad del padre, sino que es un adulto en devenir cuyos padres tienen por tarea el hacer advenir sus cualidades profundas.

Las formas en que las mujeres analizadas en este estudio optan por vivir sus embarazos y partos son indisociables de la reflexión ligada al tipo de crianza que desean para sus hijos e hijas ${ }^{8}$. Bajo el mismo imperativo del «respeto de los tiempos naturales de cada persona», definen su estilo de crianza como «natural» o «de apego», que concibe al niño como un ser distinto de los padres, con inquietudes, tiempos y deseos propios. Este estilo de crianza prioriza los lazos afectivos ligados a la familia y al cuidado y se propone recuperar lo que ellas perciben como «natural» mediante una serie de prácticas que a continuación describiremos.

La noción de crianza que acuñan las mujeres de este estudio se traduce en términos sociológicos en la noción de «cuidado de los niños pequeños». En palabras de Hochschild, esta refiere a «un vínculo emocional, usualmente recíproco, entre la persona que brinda el cuidado y la que lo recibe. En el marco de ese vínculo, la persona que brinda el cuidado se siente responsable por el bienestar de otros y lleva a cabo un trabajo mental, emocional y físico con el fin de cumplir con esa responsabilidad. Por consiguiente, cuidar a una persona implica

situarse en el siglo XVII, los bebés comienzan a ser amados como individuos en lugar de ser mimados como pequeños animales, y los niños pequeños ya no son tratados como adultos en miniatura sino como individuos con necesidades específicas. En cierta forma, en un determinado momento, la sociedad habría literalmente «inventado» al niño pequeño y luego al adolescente.

6 En el ensayo La civilización de los padres (1998), Elias se vale del análisis histórico y la perspectiva a largo plazo para dar cuenta de un proceso de transición de relaciones más autoritarias entre padres e hijos a relaciones más igualitarias.

7 A partir de la Declaración de los Derechos del Niño (1959), de la Convención Internacional sobre los Derechos el Niño (1989) y de la labor de organismos internacionales como Unicef y Unesco.

8 Mencionamos la cuestión del embarazo y parto ya que el tema abordado en el artículo se enmarca en una investigación doctoral más amplia que se propone indagar, describir y comprender las formas en las que construyen su maternidad mujeres de la ciudad de Santa Fe (Argentina) que participan de grupos de reflexión sobre el embarazo, parto y crianza. Muchas de las prácticas y significados asociados con la maternidad que se construyen al interior de dichos grupos de mujeres se acerca a la caracterización que hace Anna Fedele (2016) de la «maternidad holística», en la cual el embarazo, el parto y los cuidados en la primera infancia son considerados como importantes eventos espirituales en la vida de la madre y el niño o la niña. 
interesarse por ella» (Hochschild, 2008, p. 309). En su mayor parte, el cuidado requiere un desempeño tan personal, tan imbuido de sentimientos, que rara vez es percibido por quienes lo ejercen como trabajo - a menos que forme parte de un contrato de trabajo a partir del cual se obtiene una remuneración-. De allí que las feministas construyeran las categorías de «trabajo reproductivo» y «trabajo no remunerado» con el fin de cuestionar el paradigma según el cual el ámbito privado es un espacio en el que no se «produce» nada, entendiendo al cuidado como el trabajo necesario para la reproducción de la vida. Partimos de la idea de que el cuidado comprende actividades tanto prácticas como simbólicas, lo cual nos lleva a recuperar el valor que supone realizar las tareas de cuidado, así como también el derecho de las personas — en este caso, niños - a recibir cuidados.

Cuando se pregunta a las entrevistadas sobre el cuidado de sus hijos, responden con una reflexividad que merece nuestra atención. Ellas comentan llevar adelante un «estilo de crianza» particular, conversan con sus parejas sobre la manera de criar a sus hijos, consumen literatura vinculada con la temática y participan de talleres y espacios de reflexión con otras madres, padres y expertos. De la misma forma en que hablan y reflexionan sobre sus propios embarazos y partos, cuando se refieren a la crianza de sus hijos lo hacen con un nivel de información y conocimientos específicos que merece atención. El cuidado y la vida cotidiana de sus pequeños hijos emerge en las entrevistas como una temática que pueden explicar y justificar con precisión.

Nosotros seguimos el estilo de crianza natural, de tomar todas las herramientas naturales de la crianza, el colecho, la teta. Va a tener cuatro años y sigue con la teta. El tema de la pedagogía positiva, de ayudarlos a construir su autoestima y, en relación a los límites es imposible no ponerles, pero los límites que uno marca no son arbitrarios, siempre va a haber explicaciones (fragmento entrevista Luz).

Desde la propia perspectiva de las mujeres estudiadas, la crianza no consiste en un asunto fácil, cotidiano que cualquier madre o padre puede llevar a cabo, sino en un tema complejo que lleva aparejada la necesidad de conocimientos, aptitudes especiales y conciencia de la complejidad.

En las ciencias sociales existe una vasta literatura caracterizada por cierta actitud de «sospecha» acerca de la arbitrariedad de los consejos dados por expertos a las mujeres. A partir de dicha bibliografía, se impone una razonable desconfianza sobre las certezas que guían las prácticas vinculadas con la crianza en la actualidad y también sobre el éxito de consumo que las acompañan. Como bien señala Darré (2013): 
Con excepción de unos pocos consejos pausterianos a fines del siglo XIX, la arbitrariedad de los consejos dados por los especialistas es un dato que se destaca aún en la actualidad. El jugo de naranja ha sido beneficioso en algunos períodos y peligrosísimos en otros [...]. En la misma línea, el sentido del eczema, de la falta de apetito o de enuresis nocturna ha cobrado significados diferentes, incluso ha aparecido o desaparecido del horizonte de los problemas infantiles y de la culpa materna (p. 196).

Las prácticas maternales de cuidado asociadas con el estilo de crianza natural se refuerzan de manera continua en los talleres y encuentros de crianza y lactancia. Mediante la socialización de un determinado repertorio de prácticas maternales de cuidado, activamente contribuyen a la construcción de una subjetividad como madres, marcando un límite entre las «buenas» y «malas» prácticas de cuidado, convirtiéndose las madres en objeto de atención y control de la mano de consejos de otras madres «experimentadas» y doulas, ${ }^{9}$ de la psicología de divulgación como Laura Gutman ${ }^{10}$ — verdadera guía espiritual de madres puérperas — , de médicos expertos en la temática — como el obstetra Michel Odent ${ }^{11}$ y el pediatra Carlos Gonzales $^{12}$ - y de recomendaciones de organizaciones internacionales como la Organización Mundial de la Salud y la Liga de la Leche.

9 La doula cumple un rol fundamental como acompañante de la mujer-madre en el proceso de embarazo y parto, y como socializadora de prácticas maternales vinculadas con el cuidado de los recién nacidos. Si bien no tienen una formación académica formal, en los distintos espacios de formación que se ofrecen adquieren conocimientos básicos sobre físiología del embarazo, parto y puerperio, primeros auxilios, puericultura, lactancia y educación prenatal (Felitti y Abdala, 2018).

10 Laura Gutman es psicoterapeuta familiar especializada en la atención de madres de niños pequeños y parejas. Graduada como psicopedagoga clínica en París, fue discípula de Françoise Dolto. Se especializó en las temáticas de maternidad, lactancia y vínculos familiares. Fundó y dirige Crianza, institución de Buenos Aires donde funciona una escuela de capacitación para profesionales de la salud y la educación, los grupos de crianza para madres, un equipo de doulas a domicilio para mujeres puérperas, terapias individuales y de pareja y publicaciones sobre maternidad y crianza. http://www.lauragutman.com.ar/

11 Michel Odent es un prestigioso ginecólogo francés, pionero y referente de la promoción del parto fisiológico, precursor de los partos en el agua, y de las teorías que plantean el beneficio de incentivar la lactancia materna durante la primera hora del nacimiento. Entre sus obras más destacadas se encuentran La cientificación del amor (2001), El granjero y el obstetra (2002), La cesárea: ¿problema o solución (2006), El bebé es un mamífero (2009) y El nacimiento en la era del plástico (2011).

12 Carlos Gonzales es un médico pediatra español y autor de varios libros sobre crianza, alimentación y salud infantil. Entre sus obras más destacadas se encuentran Mi niño no me come (1999), Bésame mucho, cómo criar a tus hijos con amor (2003), Manual práctico de la lactancia materna (2004), Un regalo para toda la vida, guía de la lactancia materna (2006), Comer, amar, mamar (2009) y Creciendo juntos (2013). A su vez, es fundador y presidente de 
La lógica con la que opera la socialización de las «buenas» prácticas de cuidado mediante recomendaciones y consejos es la siguiente: primero se enumeran los beneficios de llevar adelante esas recomendaciones (beneficios de tipo biofisiológicos, psicológicos, ecológicos y espirituales); luego aclaran que «no son prescripciones normativas», sino solo «recomendaciones» que pueden tomarse solo si lo «quiere» y lo «siente», para al final alertar sobre los riesgos o peligros que puede acarrear no llevar adelante dichos consejos.

Lo que decimos acá es que la crianza forma parte de la vida privada de una familia. No tiene que estar diciéndote nadie si le ponés un pañal de tela, descartable o si lo dejás en pata, porque es una decisión de la familia. Pero cuando hablamos de crianza natural es difícil no asociarla a las necesidades de la criatura. Cuando no se satisfacen sus necesidades primarias, puede sufrir un alto nivel de estrés (esto incluye desde un nacimiento traumático, la separación de su madre al nacer, poco contacto físico, obligarla a dormir sola aunque llore, etc.). Este estrés puede llevar al desarrollo de cierto sentimiento de aislamiento y desconfianza que puede acompañar durante muchos años en la infancia (miedo a la oscuridad, por ejemplo), adolescencia y edad adulta (personas inseguras de sí mismas) (registros de conversaciones, taller de preparto).

A continuación enumeramos una serie de prácticas de cuidado que se socializan y aprenden en los talleres, identificadas a partir de las observaciones y entrevistas realizadas.

\section{Unión piel con piel con el bebé desde el nacimiento}

En los cursos de preparto resaltan con frecuencia la importancia de unos de los pilares de la crianza natural: el contacto «piel con piel» del bebé con su madre. En este sentido, promueven que el recién nacido sea colocado en el pecho de su madre, para darle inmediatamente de mamar.

Los bebés están todo el día pegados a su mamá; ellos necesitan estar seguros y contenidos. Y que estén pegados a su cuerpo todo el día es beneficioso para la madre: es más fácil para entender al bebé, sus llantos, muecas sonidos. Aprender a decodificar el mensaje de los bebés es más fácil si tu bebé está pegado a vos durante el día. Chicas, recién se están conociendo, hay que crear el vínculo (notas conversaciones, taller de lactancia).

la Asociación Catalana Pro Lactancia Materna y Miembro del Consejo de Asesores de Salud de La Leche League International. 
Desde esta perspectiva, existiría una «necesidad biológica y psicológica» de la madre de estar en contacto con el bebé inmediatamente después del nacimiento para poder establecer entre ambos un «vínculo satisfactorio». Desde esta premisa se derivan dos prácticas: el «porteo» y el «colecho».

Algo que llamaba nuestra atención en los talleres de crianza era la entrada de las mujeres al salón: llegaban al taller luciendo a sus niños cargados en sus pechos o espaldas. Como el contacto físico durante los primeros meses hasta el primer año es considerado por estos grupos como la condición para que «la criatura se críe en un clima de seguridad, amor y respeto», las madres se sienten interpeladas a asistir a los talleres en los que se les informe sobre los diversos modelos y posiciones para colgar al bebé. En un taller de crianza se trabajó exclusivamente sobre los distintos modelos de portabebés (fular elástico, mochila ergonómica, rebozo, meitai, sling) y las diferentes posiciones para colocar a los niños, llamándonos la atención sobre toda la «ingeniería» y mercado desarrollado sobre el porteo. El cochecito - verdadero objeto profano - no tiene lugar en estos espacios. Los beneficios asociados con el porteo los siguientes:

Los bebés que son llevados en brazos o en portabebés están al resguardo. Se sienten más protegidos, se estresan menos y permanecen en un estado de vigilia tranquila, lo cual les permite asimilar mejor lo que sucede en su entorno. La cercanía y el contacto continuo con el bebé permiten conocerlo mejor fortaleciendo el vínculo; los bebés que están más tiempo en posición horizontal llegan a la verticalidad con más recursos motores (registro conversación, encuentro de crianza Tinkunako).

Otro de los pilares que forman parte del continuum de prácticas asociadas a fomentar un vínculo «piel con piel» es dormir con el bebé.

Dormir acompañado evita la angustia de separación que experimentan algunos niños durante la noche, haciendo que duerman más y descansen mejor. Ayuda a los niños a aprender que el sueño es un momento agradable y que no está solo. En los casos en el que el bebé es amamantado, el colecho es, además, un método práctico pues tiene el pecho a su disposición en cualquier momento (registro conversación, círculo de crianza Tierra Libre).

En un taller de preparto surgió este tema cuando un padre comentó que su hermano le iba a pasar el libro Duérmete niño que tanto había ayudado a que sus sobrinos desde muy pequeños durmieran solos en su cuarto. «Es que después no lo sacás más», dice el padre, sin sospechar lo que traería aparejado su comentario. La doula se indigna y comienza a argumentar en contra de ese método de «adies- 
tramiento conductista» para que el bebé aprenda a dormir solo, el cual — según ella - consiste en «dejar al niño llorar, no acudir a su llanto, hasta que se canse y se duerma. ¿Saben lo que aprenden el bebé con esto?», increpa al padre la doula: «que no cuenta con sus padres», sentencia.

\section{Poner límites de manera «amorosa»}

La concepción de los niños como sujetos de derecho y el principio de no violencia hacia ellos que se ha institucionalizado en nuestras sociedades contemporáneas (Carli, 1999) se manifiestan concretamente en esta pedagogía maternal en la manera en que las familias reflexionan con respecto a la forma de poner límites a sus hijos:

Otro pilar se relaciona con la disciplina que uno tiene que impartir; así como respetás sus tiempos, sus llamadas, sus necesidades, también la educación parte de la premisa de no castigo y de guiar en lugar de condicionar el comportamiento. Podés decirle sin tener que castigar qué es lo que está bien y qué es lo que está mal, y sin reprimir y sin someter al niño que es el más débil. Culturalmente estamos acostumbrados a eso, al sometimiento del más débil. Y también para nosotros es un gran aprendizaje este modelo, porque se supone que también estamos aprendiendo de ellos (fragmento entrevista Josefina).

En los círculos de crianza, las mujeres conversan mucho sobre lo cansadas que se sienten a veces y los riesgos que corren de «desbordar» y tratar «mal» a sus hijos. Las mismas doulas comentan que lo más importante de los círculos de crianza es que las mujeres conversen y hablen sobre los propios miedos, frustraciones y contradicciones que sienten, pues los talleres funcionan como espacios de «catarsis».

Es complicado, sobre todo cuando estás cansado. A mí a veces me encuentro diciéndole «si no hacés tal cosa no vamos a jugar...». Y bueno, es complicado, pero tiene sus frutos, en el sentido de que Dante es bastante empático e intenta siempre expresar lo que siente. Errores cometemos siempre. En este estilo de crianza nosotros optamos mucho por pedir perdón (fragmento entrevista Luz).

El no utilizar «métodos de adiestramiento» basados en la «violencia y manipulación» les genera muchos problemas y discusiones con sus propios padres y - en algunos casos - parejas, manifestando las diferencias entre cómo ellas fueron criadas y las formas en que ensayan la crianza de sus hijos. 
Para mí un «no» de mis viejos [padres] era palabra santa y seguramente me enseñaron el «no» de una forma muy ruda. Yo no quiero eso para mi hija. A ver, no quiero que meta los dedos en el enchufe, pero se lo tengo que decir con un «no» amoroso para que lo entienda. Eso es lo que tenemos que hacer, poner los límites de manera amorosa. Antes era un «no y andáte, calláte». Ahora tratamos de explicarle por qué no, de desviarle la atención. Creo que la crianza hoy es mucho más amorosa, se da más libertad al niño para que se desarrolle (fragmento entrevista Alejandra).

\section{Lactancia materna}

Incentivar la lactancia materna exclusiva (sin ningún tipo de complemento, al menos hasta los seis meses) y a libre demanda (no pautar horarios ni rutinas, sino dar de mamar cuando el bebé lo desee) es la gran batalla que se propone ganar el estilo de crianza natural. Si el objeto no deseado para incentivar el porteo es el cochecito, en este caso lo son la mamadera y el chupete. Asimismo, es en la práctica de la lactancia materna donde los riesgos y peligros se tornan más problemáticos y alarmantes si no se lleva a cabo.

No existe sustituto para la leche materna. Las leches artificiales están preparadas a partir de leche de vaca y contienen solo una parte de los nutrientes y ninguna de las hormonas y los anticuerpos que están en la leche humana. Existen numerosos riesgos asociados a la leche artificial: mayor riesgo de asma, alergias, infecciones de las vías respiratorias y gastrointestinales, digestivas, oclusión dental alterada, infección por contaminación de la leche artificial, carencias de nutrientes, enfermedades crónicas a lo largo de la vida, como diabetes tipo I, celiaquía, algunos tipos de cáncer, enfermedades intestinales inflamatorias, obesidad, otitis media e infecciones del oído. Aporte inmunológico/desarrollo del cerebro (fragmento de un texto compartido en Facebook de una doula a las madres).

Pero los beneficios de la lactancia materna no se agotan en los de tipo biofisiológico: beneficios de índoles espirituales y psicológicos también son asociados a esta práctica, dejando a quienes no dan la teta en un lugar bastante peculiar entre lo exótico y lo abyecto.

Amamantar es mucho más que dar el mejor alimento: también es contacto, consuelo, comunicación (fragmento entrevista Claudia).

La lactancia materna favorece y fortalece la relación de afecto entre la mamá y el bebé y contribuye al desarrollo de niños capaces, seguros y emocionalmente estables (Guía del Ministerio de Salud y Desarrollo Social de la República Argentina). 
«Dar la teta es dar lo mejor de vos»; «La lactancia es el vínculo de amor que vuelve sanos, fuertes y felices a nuestros hijos» (registro de conversaciones, círculo de lactancia).

El mensaje es claro: la buena madre es aquella que amamanta.

En efecto, hemos visto cómo las fronteras entre prácticas de crianzas apropiadas e inapropiadas son sistemáticamente marcadas, y cómo el «sentir» y el «querer» atraviesan por completo la argumentación ligando dichas prácticas de cuidado al placer y al amor.

\section{¿QUIÉN CUIDA A LOS NIÑOS? SITUACIONES TÍPICAS IDEALES DE CUIDADO: ENTRE LA FEMINIZACIÓN Y COMUNITARIZACIÓN DE LAS CRIANZAS}

Como hemos analizado, las mujeres estudiadas parten de una concepción de crianza que - según el continuum de prácticas descritas - supone un «modelo de maternidad intensiva» (Hays, 1998), que descansa en una visión de los niños como seres valiosos e inocentes, en donde la crianza debe ser llevada a cabo ante todo por madres que deben prestar cuidado y atención intensiva a sus hijos, mediante una alta dosis de entrega y disposición emocional y corporal. De esta forma, el preguntarnos sobre quién cuida a los niños nos condujo a indagar acerca del modo en que las mujeres y sus familias se organizan con el cuidado, identificando - a través del trabajo de campo- dos situaciones típicas de cuidado: las madres cuidadoras de tiempo completo y la comunitarización de la crianza. El análisis se enfoca en las elecciones sobre el cuidado de las mujeres estudiadas y sus familias. Retomando a Faur (2014), las decisiones con relación a la organización del cuidado de los niños por las familias responden básicamente a dos procesos: la relación - y tensión - entre la oferta de servicios que permiten desfamiliarizar el cuidado y su demanda, y las negociaciones de género al interior de los hogares.

Las mujeres de este estudio poseen en su mayoría títulos universitarios o se encuentran estudiando alguna carrera universitaria, pero - con excepción de las dos mujeres que tienen hijos más grandes - no trabajaban en su campo profesional al momento de la entrevista. En efecto, el rasgo característico de las mujeres estudiadas es la retirada por un tiempo del mercado laboral (más del estipulado por licencia por maternidad) luego de tener a sus hijos. Es decir, han migrado en su mayoría del mundo del trabajo remunerado al mundo de los cuidados para dedicarse exclusivamente a la crianza de sus niños pequeños, eligiendo trabajos 
de medio tiempo o emprendiendo en sus propios hogares. En este sentido, nos hallamos frente a mujeres profesionalizadas que se preguntan a sí mismas, de manera no necesariamente explícita, «¿Realmente me siento realizada en el ámbito profesional?». Si la respuesta es afirmativa, «¿me atrevo a pensar que dedicaría toda mi vida a la profesión? ¿Verdaderamente deseo delegar el cuidado de mis hijos a otros? ¿Qué será lo mejor para mis hijos?».

Lo que observamos es que subyace una reticencia a la desfamiliarización del cuidado, resolviendo de diferentes maneras la tensión que implica para las mujeres conciliar responsabilidades y deseos con respecto a sus tiempos de dedicación a la familia y a la participación laboral, sin apelar al recurso u opción de externalizar los cuidados vía mercantilización. Una de las lógicas argumentativas que subyace a la decisión de las mujeres y sus familias de no desfamiliarizar el cuidado infantil — ya previamente trabajada — es la adhesión a los principios de la crianza natural, lo que, como vimos, demanda una alta dosis inversión temporal, emocional y corporal de la madre para con su hijo.

Ahora bien, la adhesión a estos principios de crianza converge con una argumentación «pragmática», ligada a la racionalidad económica, que indica que externalizar el cuidado de los niños pequeños supone un gasto excesivo e innecesario para el hogar. «Así no tengo que pagar a otros para que me lo cuiden» es un argumento a las que varias de las mujeres entrevistadas apelaban.

A su vez, el grupo específico de mujeres estudiado plantea la inexistencia de servicios del cuidado en la ciudad acordes a sus pautas de crianza. Las necesidades e intereses familiares vinculados con el cuidado no son contemplados en las políticas sobre cuidados vigentes, manifestando conflictos a la hora de relacionarse con servicios de cuidado tanto públicos como privados; específicamente, con una visión muy crítica hacia los jardines de infantes «tradicionales».

Tengo muchas cosas para decir en los jardines de infantes. Es peligroso, te diría, todo lo que sucede alrededor de esos niños tan chiquitos. Todas las etiquetas, los mandatos, todas las cosas que traen los maestros, los mismos niños. Es peligroso... El niño tiene que ser en ese momento solo niño en el entorno donde le tocó nacer y desarrollarse en esos primeros años de vida como se le antoje, y no con una asignatura que le diga cómo hacer, cómo dirigirse a los otros, y rutinas que son extra a su día a día con mamá y papá, o el vínculo familiar que tenga. Me parece que no está bueno para el niño. Hay necesidades de las mamás de separarse de los niños, lo entiendo, pero no las justifico. No me parece que tenga que ser así (fragmento entrevista Milagros). 
A través del análisis empírico, construimos dos situaciones típicas de cuidado, las cuales entendemos como respuestas a la necesidad de las mujeres y sus parejas de organizarse con relación al cuidado de los niños pequeños, respetando los deseos propios de las mujeres que, traducidos en un lenguaje sociológico, implican resistencias a la desfamiliarización y externalización del cuidado vía mercantilización. Estas situaciones de cuidado se diferencian según el grado de tensión que experimentan frente a la conciliación de las esferas del trabajo y la familia, y el nivel de feminización de los cuidados. Es necesario aclarar que estas situaciones no conforman compartimentos estancos ni estáticos, sino que se presentan como opciones dinámicas y fluctuantes. Los arreglos suelen ser transitorios, y suponen una significativa capacidad de adaptación de los involucrados. Las mujeres y sus parejas pueden cambiar de una situación a otra en función de las oportunidades que les ofrece el contexto y sus decisiones en cada coyuntura, que varían a lo largo del ciclo de la vida de los niños y la familia misma.

\section{Esquema 1. Construcción de situaciones típicas ideales de cuidado}

\begin{tabular}{clc}
\hline+ & Grado de tensión frente a la conciliación de & + \\
- & $\begin{array}{l}\text { las esferas familiar y laboral } \\
\text { Feminización de los cuidados }\end{array}$ \\
COMUNITARIZACIÓN DE LAS & - \\
CRIANZAS & MADRES CUIDADORAS DE TIEMPO \\
Tierra Libre & COMPLETO \\
& \\
\hline
\end{tabular}

\section{Las madres cuidadoras de tiempo completo: la feminización de los cuidados en clave de deseo}

Caracterizamos a esta situación típica de cuidado como aquella en la cual las mujeres sostienen una situación de cuidado en la que su presencia, de tiempo completo, forma parte de los arreglos domésticos vigentes. Es necesario resaltar que, en los casos que entrevistamos, las madres de tiempo completo son mujeres que tuvieron experiencias de trabajos remunerados vinculados con su formación profesional.

Antes de estar embarazada y durante el embarazo estaba trabajando como becaria doctoral. Y justo cuando decidí renunciar, me entero de que estaba embarazada y dije «chau, qué loco». Y por un lado, en cuanto a estar con ella 
me vino espectacular porque todo este año estuve ocupándome de la bebé, todo el tiempo con ella (fragmento entrevista Guillermina).

Como que nunca asocié la docencia con la realización personal. También quería entregarme a ser mamá. Fue eso. Estaba claro que no quería volver, pero costaba soltar. Nada, terminé las vacaciones y renuncié. Y todos me miraban como «la loca renuncia para estar con el bebé» (fragmento entrevista Florencia).

De esta forma, quedarse en casa no se presenta como un destino inexorable y unívoco, y mucho menos como una decisión que puede perdurar toda la vida; tampoco es percibido y significado por las mujeres como un obstáculo frente a su autonomía, sino todo lo contrario: lo que opera aquí es una resignificación de las tareas del cuidado. Frente a los discursos que ellas dicen que las interpelan como mujeres de clases medias profesionales en las que se asocia lo doméstico a la subordinación femenina, a la falta de autonomía y de reconocimiento, ellas reconvierten y se reapropian del espacio doméstico como un ámbito donde es posible realizarse personalmente. En la misma clave que desean «recuperar» sus partos, el «regreso al hogar» es un acto ofensivo frente a lo que ellas conciben como la expropiación de la experiencia de la maternidad.

Creo que es lo mejor para mi hija y para mí que me quede con ella. De hecho, hoy en día varias mamás con las que me cruzo los han mandado al jardín y ahora tienen niñeras porque quieren que estén más en la casa [...]. Es muy difícil, porque las madres hoy necesitan salir a trabajar, pero hay mucha cuestión de que la mujer se hizo muy independiente muy profesional y quieren salir por eso. Son mujeres que tienen estudio y dicen «yo estudié ocho años, que me voy a quedar en mi casa a cuidar a mi hija, no. Me voy a trabajar, que los cuide alguien». Son elecciones, pero yo decidí esto y al día de hoy sigo firme en mi decisión (fragmento entrevista Lucía).

Es muy ilustrativa la cita de Luz, en la que cuenta que se ha sentido «engañada» por retóricas y discursos que, según ella, asocian la realización personal a la profesionalización, a costa de renunciar al ejercicio y deseo pleno de la maternidad.

Y bueno, cuando nació Dante, además de descubrir todo este nuevo mundo de la maternidad, yo me di cuenta de que me sentía un poco engañada socialmente. Primero, desde el sistema que te dice tenés que terminar de estudiar, hacer carrera y después la maternidad. Como si la maternidad fuese una carga. Y cuando él nació y tuve mi propio bebé me sentí plena, como mujer muy plena. ¡Pucha! A mí me hubiese gustado tener incluso hijos más joven y haber tenido otra cadena de eventos. Pero el hecho de toda esta postergación 
de la maternidad... Además, hay como mucho desprestigio hoy con esto de ser madre; es como que uno puede sentirse satisfecha desde un trabajo y sentir que uno se siente realizada. Como que toda una cultura de negar la maternidad y sentirse realizada desde otros aspectos. Y yo sentía que siendo mamá me sentía mucho más realizada, en eso sentía un engaño social. No me parece mal que la mujer gane este hecho de poder elegir, pero este poder elegir se transformó en una obligación, es como que se vio obligada a igualar al hombre en este sentido, negando su aspecto maternal. Y eso también hace que no se avance en todos los derechos que necesitamos como madres. Parecería que el único camino de autorrealización de la mujer está en el trabajo, como si esa fuera su condición de independencia. Y yo creo que esto tampoco es completamente cierto. ¡Pucha! Yo me siento bien, conectada, en contacto con mi femineidad como nunca antes me había sentido.

Cuando indagamos en las entrevistas sobre los recuerdos que las mujeres tienen sobre su propia infancia, confirmamos la conjetura que teníamos al comienzo de la investigación: las mujeres estudiadas se reflejan e identifican — no necesariamente de manera consciente - de manera opuesta a sus propias madres, mujeres que, según las representaciones de sus hijas, se comprometieron y encontraron una fuente de reconocimiento en el ámbito laboral, en detrimento de la inversión temporal y emocional en el cuidado de ellas y sus hermanos.

Sí, eso también me pasó con mis viejos. Ellos hasta el día de hoy no cambiaron, ellos son muy fanáticos, del deseo arraigado a lo profesional y siempre lo respetaron. Mi mamá es psicoanalista y mi papá es contador; de hecho, yo trabajo en la empresa de mi viejo. Ellos viven para eso. Eso a mí me chocó, fue muy duro... Por supuesto que no lo quiero repetir yo. De hecho, este año me lo pensé, intenté retomar la carrera al principio de año, pero dos semanas me duró. No podía, después me di cuenta de que no es lo que quería (fragmento entrevista Virginia).

Mi papá y mi mamá trabajaban todo el día; mi mamá tuvo eso de mujer moderna de esa generación. Mientras menos estabas en casa, más trabajabas, más progresista eras. Eso lo veo en general hoy también (fragmento entrevista Martina).

En fin, en un contexto en que — según la propia visión de las mujeres estudiadas - el ideal de maternidad colisiona con las obligaciones cada vez más densas del mundo del trabajo, la situación de cuidado de «madres a tiempo completo» implica no solo la incompatibilidad de las esferas laborales y familiares, sino el mismo no deseo de conciliación de las mujeres. Es decir, la feminización de los cuidados que esta situación supone significa para las mujeres una apuesta por la 
reivindicación de un repliegue hacia lo doméstico, otorgando un alto valor a los cuidados y a los afectos, sin sentir necesariamente que renuncian a su desarrollo personal e individual, sino todo lo contrario ${ }^{13}$.

\section{La comunitarización de las crianzas y la conciliación en tensión}

A diferencia de la situación de cuidado de «madres a tiempo completo», esta situación de cuidado se caracteriza por mujeres que viven con mayor tensión la conciliación entre la esfera familiar y laboral. Lo que las mujeres manifiestan en sus relatos es la incomodidad que sienten, por un lado, frente a sus deseos personales y necesidad de habitar por espacios donde pueden afirmar su autonomía más allá de la maternidad, y, por otro, el deseo y compromiso de dedicarse al cuidado de sus niños pequeños. Se hace evidente en las citas que para las mujeres de este estudio «maternar» implica postergar la realización personal frente a la existencia de un hijo.

Para mí, personalmente, es algo súper conflictivo lo que va a pasar con la gorda en unos meses. Yo necesito ejercer mi profesión y seguir estudiando. Pero es una necesidad mía. Es un negociar constante entre qué es lo que uno puede y lo que está dispuesto a relegar y qué es lo que no, así que bueno. Por ahora, yo trabajo a la tarde mediodía y él a la mañana medio día, entonces compartimos la «cuidada». Como la gorda no va al jardín, está conmigo hasta las dos, dos y media de la tarde y a partir de las tres luego él se encarga de ella (fragmento entrevista Josefina).

Las mujeres que optan por esta situación de cuidado, alrededor del año del nacimiento de su hijo o hija, comienzan a trabajar medio día y comparten el cuidado de los niños con sus parejas, algún familiar — abuela, abuelo o tía de la criatura- $\mathrm{o}$, en el caso de las mujeres entrevistadas que residen a las afueras de la ciudad, mediante la organización de un espacio de cocrianza entre familias amigas que comparten los principios de la crianza natural, llamado Tierra Libre.

Tierra Libre es, en palabras de los padres que lo organizan, «un espacio de crianza compartida que acompaña el desarrollo autónomo de los niños, basándose en el conocimiento y respeto por el movimiento libre de cada ser. Crianza consiente y compartida gran medicina para el alma y la sanación planetaria».

13 Este tipo de situaciones aparentemente atípicas o «a contrapelo» de la generalidad de las situaciones y deseos de mujeres de clases medias profesionalizadas, habilita — como plantea Segalen (2013) - a reflexionar teóricamente desde enfoques que se distancian de las visiones de la dominación y subordinación masculina, para dar lugar a tesis sobre el don gratuito y la gratificación afectiva. 
El espacio surge en el año 2014 por la inquietud de familias amigas que compartían círculos de crianza organizados a las afueras de la ciudad de Santa Fe, la mayoría de estas acompañadas por el mismo equipo de parteras y doulas en la tradición durante los embarazos y partos de sus hijos. Al momento de realizar el trabajo de campo, participaban del espacio quince familias con niños que oscilan desde el año a los tres.

Con amigos surgió la necesidad de juntarnos en círculos y hablar de crianza. Vemos que en nuestras juntadas nuestros niños se empiezan a relacionar entre ellos y confirmamos que necesitan del otro también. Entonces dijimos de armar un jardín. Jardín: un lugar donde podamos estar tomando unos mates nosotros y ellos haciendo la suya, jugando con niños de diferentes edades, para ver qué pasa, qué nos enseña. Y bueno, así fue como nace Tierra Libre. Alquilamos una casa entre todos, nos pusimos horarios para que nos sea útil. Viendo a quiénes les nace más estar con los niños, estar en la cocina, organizar los espacios, los materiales... (fragmento entrevista Lucía).

Tierra Libre constituye un ejemplo claro de la necesidad de las mujeres y sus familias de organizarse colectivamente para gestar un espacio a «imagen y semejanza» de sus deseos y expectativas vinculados con la crianza, frente a la inexistencia de ofertas de instituciones de cuidado y educativas atentas a sus necesidades.

El jardín funciona como un espacio de las familias para la reflexión conjunta sobre la crianza, como un lugar de contención y apoyo entre padres y madres. Esta situación típica de cuidado se distingue de la anterior por no apelar a la feminización de los cuidados, sino más bien a comunitarizarlos, extendiendo la crianza de los niños pequeños más allá de la familia nuclear e involucrando a familias amigas.

Tierra Libre es un espacio para compartir. Nos parece como demasiado grosa la crianza como para que cada trío o familia se haga cargo. Creemos que es mejor compartirla; que es mejor nutrirse de la experiencia de otros (fragmento entrevista Pilar).

Otro aspecto importante a señalar es que las mujeres entrevistadas, tanto las que se ubican dentro de la situación típica ideal de cuidado «madres a tiempo completo» como las de «comunitarización de las crianzas», optan por emprender trabajos de medio tiempo en el hogar, en su mayoría vinculados con el mundo de la maternidad y la crianza. En todos los talleres y espacios en los que participamos circulan bienes vendidos por las mismas mujeres: pañales de tela, portabebés, ropa para niños, copa menstrual, ropa y accesorios para la madre, etc. 
Ahora que ya es más grande, a mí me encanta poder trabajar en casa desde lo mío. Yo estoy ahora trabajando en el armado de un curso de inglés para el kínder, para una plataforma de homeschooling. De una forma fácil, para que los padres lo puedan aplicar y acompañen el aprendizaje de inglés de sus hijos. También tengo una tienda en la que vendo pañales ecológicos y portabebés ergonómicos, que son las cosas que más me interesan. Me encanta, me gusta tener un tiempo para esto también (fragmento entrevista Luz).

En ese momento descubría que podía hacer muchas cosas desde mi casa. Desde cocinar viandas hasta dar clases particulares. Es como que relajé. Ya dejé de ir a una institución a cumplir horarios. Y dije «desde ahora en adelante va a ser diferente, voy a tener otros tiempos» (fragmento entrevista Florencia).

Este tipo de emprendimientos económicos en los hogares es sintomático de un mundo laboral reglado por normas masculinas, consideradas por las mismas mujeres incompatibles con las necesidades de los niños pequeños y de quienes proveen los cuidados. Estos emprendimientos «en clave femenina» son los arreglos que encuentran algunas mujeres de este estudio para poder conciliar la maternidad con la generación de ingreso económico propio.

\section{LA PATERNIDAD DESDE LA MIRADA MATERNA}

Luego de haber efectuado la descripción de las prácticas de cuidado asociadas a la crianza natural y haber analizado las situaciones ideales de cuidado que persiguen las mujeres de este estudio y sus familias, abonamos con esta investigación a las tesis de otros trabajos que plantean que las actividades de cuidado tienen una marca genérica (Wainerman, 2005; Jelin, 2004; Rodríguez Enrique, 2015). Sin embargo, el interés específico de este apartado es arrojar luz no sobre la continuidad o la inercia del modelo tradicional de cuidado que supone desigualdades en relación con la crianza de los niños, sino más bien las miradas que las mujeres tienen sobre el lugar y la participación de los padres en la crianza ${ }^{14}$. Más allá

14 Preguntarnos sobre la igualdad de roles entre padres y madres en las tareas de cuidado supondría (ingenuamente) esperar que el modelo de cuidado que llevan adelante las familias de las mujeres de este estudio sea igualitario, de igual participación de los géneros en las tareas de cuidado de los hijos. A diferencia, la perspectiva que asumimos, no hace suposiciones sobre la igualdad ni sobre un modelo ideal final, sino que le interesa indagar el grado de alejamiento del modelo tradicional de la división del trabajo de cuidado de los hijos en este estilo de crianza en particular. 
de que sean las mujeres las destinatarias directas de los discursos pedagógicos maternales y quienes se dedican en mayor medida al cuidado de los niños, es necesario destacar que - según la mirada y los relatos de estas mujeres - la crianza de sus hijos no les pertenece exclusivamente, impugnando la idea de que la principal responsable de la crianza y el cuidado de los hijos es la mujer, e insistiendo en la necesidad de que los varones se involucren. En este sentido, la asistencia masculina ha dejado de ser un hecho extraordinario para convertirse en algo más frecuente y apreciado por las mujeres ${ }^{15}$.

Si bien, como vimos en las entrevistas y observaciones, las mujeres se valen de las experiencias de embarazo, parto y lactancia para identificar y definir la singularidad del vínculo con sus hijos, señalan la importancia del involucramiento y acompañamiento de los varones en esas mismas experiencias.

Entiendo que la maternidad es una entrega, que una tiene su cuerpo entregado a algo que te consume y después atender, despertarte y no dormir noches y meses y meses. Te involucra el cuerpo. Pero a mí me parece que eso tiene que ser compartido. A mí me hacía bien que Martín se encargue del bebé y yo poder hacer otras cosas [...]. Me parece que uno ya tiene el cuerpo involucrado nueve meses previos y otro tanto después con la lactancia. Cuando la tuve a Ema, yo laburaba en la universidad siete horas por día. Yo creo que Martín ahí se encontró con ser padre de otra forma. Yo me sacaba leche, la congelábamos y Martín le daba la leche. Y el contactarse con la necesidad del alimento, de la higiene, para mí generó otra cosa entre él y Ema. Eso se puede compartir (fragmento entrevista Alejandra).

En los cursos de preparto se estipulan encuentros en los cuales los varones deben participar, invitándolos a involucrarse activamente en el parto a través de la enseñanza de técnicas para calmar el dolor (manteos, masajes en el sacro, preparación de infusiones, etc.) y posiciones en las cuales ellos pueden ayudar a la mujer a hacer fuerza durante la fase de expulsión del bebé, informándoles sobre la posibilidad de que — si así lo desean — reciban al bebé y corten el cordón umbilical. A su vez, se habilitaba el espacio para que ellos manifiesten sus dudas, temores y expectativas en relación al parto.

15 Entendemos que abordar el lugar de varón en la crianza desde la perspectiva de las mujeres implica sesgos muy importantes si el propósito de la investigación fuese indagar los significados que los varones atribuyen a la paternidad. Sin embargo, como esta investigación no se propone ese tipo de indagación, creemos que es pertinente preguntarnos sobre la mirada materna de la paternidad. 
A través de las observaciones en los talleres, hemos visto cómo los varones comienzan a identificarse con la paternidad desde el mismo momento del embarazo de su compañera haciéndolo evidente mediante la utilización del pronombre inclusivo cuando se refieren a «nos embarazamos» o «estamos gestando». Asimismo, en los relatos de los partos, las mujeres resaltan la importancia del acompañamiento del padre durante ese momento.

Cuando las mujeres se refieren al lugar de sus parejas en la crianza de sus hijos, lo hacen contrastándolos con sus propios modelos paternos. Las mujeres evalúan y valoran el modelo de parentalidad ${ }^{16}$ que construyen con sus parejas en comparación al modelo de sus padres, resaltando y valorando la capacidad de sus parejas de «sentir» $\mathrm{y}$ «demostrar» afecto y cariño por sus hijos.

Yo veo en él y en los papás de estos niños un interés extra. Mi viejo, mi abuelo... no sé si les interesaba. Quizás que la mujer de tanto abarcarlo, ellos lo soltaban. Es importante que ellos estén activos. A mí, por ejemplo, la cosa esta de que «mamá hace las cosas de la casa, está con el bebé, está con el bebé, y papá se va a trabajar», no me va ni ahí. Bueno, yo dejé de trabajar, vos también. Bueno, hacemos cosas juntos y los dos nos encargamos de la casa, de la bebé...O sea, es todo el tiempo de a dos (fragmento entrevista Lucía).

Ahora bien, construir un modelo de parentalidad más igualitario -0 , mejor dicho, menos desigual que el de sus propios padres - supone estrategias de (re) negociación de roles, que oscilan entre el «ceder» espacio de las mujeres y el «involucrarse» de los varones. Lo que observamos es que, más allá de que en la práctica logren arribar — o no- a responsabilidades de cuidado no diferenciales por género, las mujeres y sus parejas tornan reflexiva esta problemática.

Yo creo que las mujeres se sienten como superpoderosas, o las únicas que podemos con todo. Y dejás al hombre por fuera de todo. ¿Qué lugar tienen los padres si vos no les permitís? Y después, ir siendo padres en esto de a poquito, ir en ese crecimiento de los hijos si el padre empieza desde el bebé a contener, a cuidar, a entender las necesidades del otro, que eso a los hombres les cuesta mucho más. Si desde el primer momento no se involucran, después en momentos como la adolescencia con los hijos tienen más herramientas para enfrentar esas cosas. Cómo hacés para involucrarte con un adolescente si nunca lo acariciaste, si todo te lo interpretó la madre (fragmento entrevista Florencia).

16 Por «parentalidad» entendemos un complejo sistema relacional de prácticas y modos subjetivos a través del cual los hombres y mujeres crían a sus hijos (Wainerman, 2005). 
En algunas situaciones de entrevista, las parejas de las mujeres se encontraban en la casa y, ante algunos tópicos que conversábamos, ellas sentían la necesidad de que los varones se involucrasen en la conversación. Cuando le preguntamos a Lucía sobre actividades que su hija comparte más con ella o su papá, inmediatamente ríe y se dirige a Matías de manera irónica y cómplice a la vez: «¿querés responderle vos esa pregunta y yo estoy un rato con Esme?». Lo mismo sucedió en la entrevista con Florencia, quien cuando comenzamos a abordar en la conversación el mismo tema, inmediatamente le habilita la palabra a Lichi. En ambos casos, las parejas participan del «círculo de varones», un espacio de encuentro y reflexión de padres cuyos hijos asisten a Tierra Libre.

A los fines de nuestra investigación, lo importante es señalar que las miradas que las mujeres de este estudio tienen sobre la paternidad se construyen bajo un contexto de involucramiento del varón en la crianza, que se vincula con un proceso más general de redefinición identitaria que afecta no solo a las mujeres, sino también a los varones.

\section{A MODO DE CIERRE}

En este trabajo, de carácter exploratorio y descriptivo, nos propusimos recuperar las experiencias singulares de las mujeres, otorgando relevancia a cómo ellas activamente construyen los significados y prácticas de su propia maternidad. Al mismo tiempo, nos arriesgamos a vincular el objeto con el nivel macrosocial, arriesgando interpretaciones que dan cuenta de cómo se entreteje la experiencia individual y subjetiva con la realidad histórica. Desde nuestra perspectiva, las mujeres de este estudio tornan reflexivos los contextos institucionales en los cuales se hallan inmersas, representando las formas de significar y vivenciar la maternidad una respuesta biográfica a nuevas tensiones.

Siguiendo el diagnóstico de Arlie Hochschild (2008) sobre los cambios institucionales de la familia y el trabajo, partimos de situarnos en un contexto de mercantilización de la vida íntima. Recuperando los hallazgos de nuestro estudio, sostenemos que frente a este contexto institucional, las formas de vivir la maternidad de las mujeres estudiadas y, en especial, las demandas por una «crianza natural» pueden ser comprendidas como una solución biográfica a la desfamiliarización y mercantilización del cuidado de los niños pequeños, adquiriendo una valoración positiva el retorno al hogar para el cuidado de los niños en mujeres de clases medias profesionalizadas que participan de los espacios y redes estudiados. 
En fin, lo que pretendemos con esta reflexión es registrar y recuperar las propias voces y experiencias de las madres concretas, con la finalidad de destacar que la maternidad y el vínculo entre madre-hijo no es necesariamente impuesto, sino que puede presentarse como deseado y elegido, en el interior de un contexto y espacio fuertemente regulado.

\section{REFERENCIAS}

Badinter, Elisabeth (2011). La mujer y la madre. Madrid: La Esfera Libros.

Beauvoir, Simone de (1977). El segundo sexo. Buenos Aires: Siglo XX.

Beck, Ulrich (1998). La sociedad del riesgo. Hacia una nueva modernidad. Barcelona: Paidós.

Beck, Ulrich y Elisabeth Beck-Gernsheim (2003). La individualización. Barcelona: Paidós.

Carli, Sandra (1999). La infancia como construcción social. En Sandra Carli (comp.), De la familia a la escuela. Infancia, socialización y subjetividad. Buenos Aires: Santillana.

Chodorow, Nancy (1984). El ejercicio de la maternidad. Psicoanálisis y sociología de la maternidad y paternidad en la crianza de los hijos. Barcelona: Gedisa.

Darré, Silvana (2013). Maternidad y tecnologías del género. Buenos Aires: Katz.

Elias, Norbert (1998). La civilización de los padres y otros ensayos. México, D. F.: Fondo de Cultura Económica.

Faur, Eleonor (2014). El cuidado infantil en el siglo XXI. Mujeres malabaristas en una sociedad desigual. Buenos Aires: Siglo XX.

Fedele, Anne (2016). «Holistic Mothers» or «Bad Mothers»? Challenging Biomedical Models of the Body in Portugal. Religion and Gender, Vol. 6, N. 1 (2016), 95-111. https://doi.org/10.18352/rg.10128

Felitti, Karina y Leila Abdala (2018). El parto humanizado en Argentina: activismos, espiritualidades y derechos. En Parterías de Latinoamérica. Diferentes territorios, mismas batallas. México, D. F.: Editorial del Colegio de la Frontera (ECOSUR).

Firestone, Shulamith (1970). The dialectic of sex. Londres: Jonathan Cape.

Hays, Sharon (1998). Las contradicciones culturales de la maternidad. Barcelona: Paidós. 
Hochschild, Arlie (1989). The second shift. New York: Avons Books.

Hochschild, Arlie (2008). La mercantilización de la vida íntima. Buenos Aires: Katz.

Illouz, Eva (2007). Intimidades congeladas. Las emociones en el capitalismo. Madrid: Katz. https://doi.org/10.2307/j.ctvndv74r

Illouz, Eva (2010) La salvación del alma moderna. Terapia, emociones y la cultura de autoayuda. Madrid: Katz. https://doi.org/10.2307/j.ctvm7bf7f

Irigaray, Luce (1992). Yo, tú, nosotras. Madrid: Cátedra.

Jelin, Elizabeth (2004). Pan y afectos. La transformación de las familias. Segunda edición. Buenos Aires: Fondo de Cultura Económica.

Philippe, Ariès (1987). El niño y la vida familiar en el Antiguo Régimen. Madrid: Taurus.

Rich, Adrienne (1976). Nacemos de mujer. La maternidad como institución y como experiencia. Barcelona: Noguer.

Rodríguez Enrique, Corina (2015). Economía feminista y economía del cuidado: aportes conceptuales para el estudio de la desigualdad. Nueva Sociedad, 256, 3-2015, 1-15. Fundación Foro Nueva Sociedad.

Sau, Victoria (2013). El vacío de la maternidad. Buenos Aires: Madreselva.

Segalen, Martine (2013). Sociología de la familia. Mar del Plata: Eudem.

Wainerman, Catalina (2005). La vida cotidiana en las nuevas familias. ¿Una revolución estancada? Buenos Aires: Lumiere. 